\title{
Solution of the von Mises Boundary Layer Equation Using a High-Speed Computer
}

\author{
By A. R. Mitchell
}

1. Introduction. In the problem of incompressible boundary layer flow along a semi-infinite flat plate against an adverse pressure gradient, the non-dimensional free stream velocity is taken to be

$$
u_{1}=1-x
$$

where the plate lies along the positive part of the $x$-axis. The von Mises equation for incompressible flow is, in the usual non-dimensional notation,

$$
\frac{\partial z}{\partial x}=u \frac{\partial^{2} z}{\partial \psi^{2}}
$$

where

$$
z=u_{1}^{2}-u^{2}
$$

On the plate, $\frac{\partial z}{\partial x}=-2(1-x)$ and $u$ is zero and so from (1), $\frac{\partial^{2} z}{\partial \psi^{2}}$ is infinite. This unpleasant singularity on the plate caused Görtler [1] to abandon finite difference methods of solution of the von Mises equation and it looked as if the comparative simplicity of (1) could never be utilized from the point of view of numerical solution. Thomson and the present author [2], however, showed that finite difference solutions are possible using the von Mises equation and obtained the expansion

$$
u=a \psi^{1 / 2}-\frac{2}{3 a^{2}}\left(\frac{\partial z}{\partial x}\right)_{\psi=0} \psi-\frac{7}{18 a^{5}}\left(\frac{\partial z}{\partial x}\right)_{\psi=0}^{2} \psi^{3 / 2}+d \psi^{2}+\cdots
$$

for the velocity in the vicinity of the plate, where $a$ depends only on $x$. This expression for $u$ incorporates the conditions of compatibility at the plate and provides a means of obtaining a value of the skin friction on the plate from the computed values of the velocity in the boundary layer. The skin friction is given by the value of $-\frac{1}{2} \frac{\partial z}{\partial \psi}$ on the plate, which from (2) is equal to $\frac{1}{2} a^{2}$.

The calculation in [2], however, was carried out on a desk computer and its extent was limited, particularly in the vicinity of the plate. In the present paper it is hoped to remedy this defect by using a finite difference form of the von Mises equation which is particularly suitable for exploring the region near the plate, and carrying out the calculation on an automatic computer.

2. The Finite Difference Equation. The four-point explicit difference replacement of (1) with $\psi=r \ell$ and $x=s h$ is

$$
z_{r, 8+1}=A z_{r+1, s}+B z_{r, s}+C z_{r-1, s}
$$

Received November 21, 1960; revised February 10, 1961 
where $A=C=\delta u_{r, s}$, and $B=1-2 \delta u_{r, s}$, with $\delta=h / \ell^{2}$, where $h$ and $\ell$ are the non-dimensional mesh lengths in the $x$ - and $\psi$-directions respectively. Since $A+B+C=1, A>0, C>0$, it follows from Richtmyer [3] that $z$ is bounded provided $B>0$, which leads to

$$
\delta<\frac{1}{2 u_{r, s}} .
$$

This is a very satisfactory condition, since it means that in the region next to the plate where $u$ is small, $h$ can be chosen correspondingly large for a given value of $\ell$. From the point of view of computation, however, it is much better to keep $h$ constant, and so $\ell$ can be reduced in the vicinity of the plate. This enables the region next to the plate to be examined in greater detail than the rest of the field.

With this in view, a distribution of nodes in the $\psi$-direction is chosen according to the formula

$$
\psi_{r}=\frac{1}{2} r(r+1) \ell_{0} \quad(r=0,1,2,3, \cdots 20) .
$$

In the $x$-direction, the nodes are given by

$$
x_{s}=s h \quad(s=0,1,2,3, \cdots) .
$$

The four-point forward difference replacement of (1) is now

$$
z_{r, 8+1}=z_{r, 8}+u_{r, 8} \delta \frac{r z_{r+1,8}+(r+1) z_{r-1,8}-2\left(r+\frac{1}{2}\right) z_{r, 8}}{r\left(r+\frac{1}{2}\right)(r+1)}
$$

where $\delta=h / \ell_{0}^{2}$ and (4) can be rearranged to give

$$
z_{r, 8+1}=D z_{r+1, s}+E z_{r, 8}+F z_{r-1,8}
$$

where

$$
\begin{aligned}
& D=\frac{\delta u_{r, 8}}{\left(r+\frac{1}{2}\right)(r+1)}>0 \\
& E=1-\frac{2 \delta u_{r, 8}}{r(r+1)} \\
& F=\frac{\delta u_{r, 8}}{r\left(r+\frac{1}{2}\right)}>0
\end{aligned}
$$

and $D+E+F=1$. If

$$
\delta<\frac{r(r+1)}{2 u_{r, s}}
$$

it follows that $E>0$, and so the theoretical solution of the difference equation (4) is bounded. However, in view of the non-linear nature of the difference equation, condition (5) does not necessarily guarantee the boundedness of the numerical solution of (4), and a watchful eye should be kept on the calculation in case any instability arises.

The principal part of the truncation error in (4) is given by

$$
\frac{1}{2} h^{2} \frac{\partial^{2} z}{\partial x^{2}}-\frac{1}{3} u \delta \ell_{0}^{3} \frac{\partial^{3} z}{\partial \psi^{3}} \text {. }
$$


In the vicinity of the plate, from (2),

$$
\frac{\partial^{3} z}{\partial \psi^{3}} \doteqdot \frac{1}{a}(1-x) \psi^{-3 / 2}
$$

and so the truncation error at nodes near the plate becomes approximately

$$
-\frac{1}{3}(1-x) h \text {. }
$$

Thus, although $\frac{\partial^{3} z}{\partial \psi^{3}}$ and higher derivatives may be very large in the neighborhood of the plate, the truncation error can in fact be small if $h$ is small.

3. The Calculation. In the calculation carried out in the present paper, the starting values of $z$ at $x=0.05$ are obtained from Howarth [4] after a certain amount of computation. The values to eight places of decimals are given in Table I. The mesh lengths are $h=0.0005$ and $\ell_{0}=0.01$, and so $\delta=5$. It can be seen from the last column of Table I that the inequality (5) is satisfied for all $r$ at the start of the computation. Since the values of $u$ decrease subsequently at nodes in the same row, there is no danger of condition (5) being violated anywhere in the field.

The calculation was carried out explicitly using (4) on a DATATron 205 highspeed computer, and values of $z$ obtained were rounded off after eight places of decimals. The machine was allowed to run until a value of $z$ was obtained on $\psi=0.01$ which exceeded the boundary value on $\psi=0$ at the same station $x$. The machine was then confronted with the task of taking the square root of a negative quantity, and so ceased to print out. This actually occurred at $x=0.137$. Thus,

TABLE I

\begin{tabular}{r|c|c|c|r}
\hline$r$ & & & & \\
& & & & $r(r+1)$ \\
0 & 0 & & & \\
1 & 0.01 & 0.1480 & 0.90250000 & \\
2 & 0.03 & 0.2620 & 0.88059600 & 6.76 \\
3 & 0.06 & 0.3726 & 0.83385600 & 11.45 \\
4 & 0.10 & 0.4846 & 0.76366924 & 16.10 \\
5 & 0.15 & 0.5910 & 0.66766284 & 20.64 \\
6 & 0.21 & 0.6845 & 0.43395975 & 25.38 \\
7 & 0.28 & 0.7673 & 0.31375071 & 30.68 \\
8 & 0.36 & 0.8337 & 0.20744431 & 36.49 \\
9 & 0.45 & 0.8831 & 0.12263439 & 43.18 \\
10 & 0.55 & 0.9165 & 0.06252775 & 50.96 \\
11 & 0.66 & 0.9355 & 0.02733975 & 60.01 \\
12 & 0.78 & 0.9452 & 0.00909696 & 70.55 \\
13 & 0.91 & 0.9487 & 0.00246831 & 82.52 \\
14 & 1.05 & 0.9499 & 0.00018999 & 95.92 \\
15 & 1.20 & 0.9500 & 0.00000000 & 110.54 \\
16 & 1.36 & 0.9500 & 0.00000000 & 126.32 \\
17 & 1.53 & 0.9500 & 0.00000000 & 143.16 \\
18 & 1.71 & 0.9500 & 0.00000000 & 161.05 \\
19 & 1.90 & 0.9500 & 0.00000000 & 180.00 \\
20 & 2.10 & 0.9500 & 0.00000000 & 200.00 \\
& & & & 221.05 \\
\hline
\end{tabular}


TABLE II

\begin{tabular}{c|c|c}
\hline$x$ & $z$ & $u$ \\
\hline 0.0500 & 0.88059600 & 0.1480 \\
0.0600 & 0.86538596 & 0.1349 \\
0.0700 & 0.84951282 & 0.1244 \\
0.0800 & 0.83344382 & 0.1125 \\
0.0900 & 0.81730237 & 0.1039 \\
0.1000 & 0.80118136 & 0.0939 \\
0.1100 & 0.78516342 & 0.0833 \\
0.1200 & 0.76935383 & 0.0713 \\
0.1250 & 0.76158997 & 0.0635 \\
0.1300 & 0.75401304 & 0.0537 \\
0.1350 & 0.74695089 & 0.0357 \\
0.1355 & 0.74632746 & 0.0321 \\
0.1360 & 0.74574286 & 0.0274 \\
0.1365 & 0.74521891 & 0.0203 \\
\hline
\end{tabular}

values of $z$ were obtained in the field from $x=0.05$ to $x=0.1365$ at intervals of 0.0005 for twenty values of $\psi$ between $\psi=0$ and $\psi=2.10$. The values of $z$, together with the corresponding values of $u$ obtained at $\psi=0.01$, are shown in Table II for several stations $x$. The actual running time of the machine was determined by the time required to print out the results. When only the values of $z$ at $\psi=0$ and $\psi=0.01$ were printed out, the machine took only four minutes for the entire run. This gives a good indication of the simplicity of the present scheme for solving the boundary layer equation.

4. Comparison of Results. Accuracy must not be sacrificed for speed and simplicity of calculation, however, so the results are now compared with those obtained by Leigh [5], who solved the boundary layer equations numerically with $u$ as a function of $x$ and $y$, using the approximation of Hartree and Womersley [6]. It is sufficient to say that the results in the present calculation at $x=0.12$ agree with those of Leigh at $x=0.1198$ to within 0.5 per cent. For example, at $\psi=0.01$ $x=0.12$, the node in the present calculation where the disagreement is likely to be greatest, the value of $u$ is 0.0713 , compared with a value of $u$ between 0.071 and 0.072 from Leigh at $\psi=0.01, x=0.1198$. It is unwise to quote Leigh's results more accurately at the nodes of the present calculation, as numerical integration and interpolation are necessary to obtain them from his original calculation. The close agreement is very encouraging considering the vastly different natures of the two calculations. Leigh started his calculation at $x=0.10$ with values taken from Hartree [7], whereas the present calculation commenced at $x=0.05$ with values taken from Howarth. Also, Leigh solved a set of simultaneous linear equations at each step $x$, whereas the present calculation involves a simple explicit calculation at each node.

5. The Singular Solution. Returning to the calculation in this paper, it is interesting to examine the solution in the neighborhood of the breakdown station $x_{b}$ which is somewhere between $x=0.1365$ and $x=0.1370$. By plotting $\log \left[-\frac{1}{2}\left(\frac{\Delta z}{\Delta \psi}\right)_{\psi=0}\right]$ 
against $\log \left(x_{b}-x\right)$ for different values of $x_{b}$, it is found that

$$
\left[-\frac{1}{2}\left(\frac{\Delta z}{\Delta \psi}\right)_{\psi=0}\right] \alpha\left(x_{b}-x\right)^{q}
$$

where the corresponding values of $x_{b}$ and $q$ are

$\begin{array}{lllll}x_{b} & 0.1369 & 0.1368 & 0.1367 & 0.1366 \\ q & 0.73 & 0.67 & 0.60 & 0.52\end{array}$

The values of $x$ considered for each $x_{b}$ are $0.1365,0.1360,0.1355,0.1350$, and at each of these stations $\left(\frac{\Delta z}{\Delta \psi}\right)_{\psi=0}$ is given by

$$
\left(\frac{\Delta z}{\Delta \psi}\right)_{\psi=0}=\frac{z_{\psi=0.01}-z_{\psi=0}}{0.01}
$$

Despite the fact that the above result is based on a breakdown point in a finite difference calculation, there is a distinct resemblance between it and the result

$$
\left(\frac{\partial u}{\partial y}\right)_{y=0} \alpha\left(x_{s}-x\right)^{\frac{1}{2}}
$$

obtained by Goldstein [8] from the asymptotic solution of the differential equation valid in the neighborhood of the separation point, where $x_{s}$ is the separation point, and $\left(\frac{\partial u}{\partial y}\right)_{y=0}$ is the skin friction in the physical $(x, y)$ plane. Consequently, it is felt that further finite difference calculations using the von Mises variables may go a long way towards determining the nature of the solution of the boundary layer equation in the neighborhood of the separation point.

6. Acknowledgements. The author is indebted to John Todd and Joel Franklin of the Mathematics Department, California Institute of Technology, for helpful discussions on topics related to the present paper, and also to Ken Hebert for carrying out the numerical work on the DATATRON 205. Thanks are also due to the Office of Naval Research for financing the present project during the author's stay in California.

St. Andrews University

Fife, Scotland

1. H. GörTLER, "Weiterentwicklung eines Grenzschichtprofiles bei gegebenen Druckverlauf," Z. Angew. Math. Mech., v. 19, 1939, p. 129-141.

2. A. R. Mitchell \& J. Y. Thomson, "Finite difference methods of solution of the von Mises boundary layer equation, with special reference to conditions near a singularity," $Z$. Angew. Math. Phys. v. 9, 1958, p. 26-37.

3. R. D. Richtmyer, Difference Methods for Initial-Value Problems, Interscience Publishers, New York, 1957, p. 13 .

4. L. How ARTH, "On the solution of the laminar boundary layer equations," Proc. Roy. Soc. London Series A, v. 164, 1938, p. 547-579.

5. D. C. F. LEIGH, "The laminar boundary-layer equation: a method of solution by means of an automatic computer," Proc. Cambridge Philos. Soc., v. 51, 1955, p. 320-332.

6. D. R. HARTREe \& J. R. Womersley, "A method for the numerical or mechanical solution of certain types of partial differential equations," Proc. Roy. Soc. London Series A, v. 161, 1937, p. 353-366.

7. D. R. HARTREe, "A solution of the laminar boundary layer equation for retarded flow," Aero. Res. Council, Rep. and Memo. No. 2426, 1949.

8. S. Goldstein, "On laminar boundary-layer flow near a position of separation," Quart.J. Mech. Appl. Math., v. 1, 1948, p. 43-69. 